\title{
CLIMATÉRIO: SINTOMATOLOGIA VIVENCIADA POR MULHERES ATENDIDAS NA ATENÇÃO PRIMÁRIA
}

\author{
*Rita de Cássia Alves Peixotol \\ Taysa de Sousa Tolentino " \\ Willames da Silva! \\ Adelson Francisco Ferreira III \\ Edna Samara Ribeiro César IV \\ Estela Rodrigues Paiva Alves ${ }^{V}$
}

\section{RESUMO}

O climatério é uma fase que ocorre durante o ciclo vital da mulher, acompanhada por um conjunto de transformações fisiológicas, sociais e psicológicas. Representa o marco do envelhecimento feminino, influenciando consideravelmente na qualidade de vida por possuir um complexo de sinais e sintomas específicos. A pesquisa objetivou identificar a sintomatologia vivenciada por mulheres no período do climatério, atendidas na atenção primária. Para isso, foi realizado um estudo exploratório e descritivo, com abordagem quantitativa, realizado em uma Unidade da Estratégia Saúde da Família, no município de João Pessoa/PB, tomando como amostra 50 mulheres com idades entre 40 e 65 anos. Avaliaram-se as características sociodemográficas e utilizou-se o Índice Menopausal de Blatt \& Kuperman (IBMK), que é capaz de identificar os sinais e sintomas do climatério e classificá-los conforme a intensidade: leve, moderado e intenso. Conclui-se que, das mulheres entrevistadas, $52 \%$ encontravam-se com idade inferior a 50 anos e $44 \%$ frequentaram a escola durante 9 anos. Com relação à intensidade dos sintomas do climatério, percebe-se que $48 \%$ apresentaram manifestações leves. Houve predominância de palpitações para leves (40\%), cefaleia para moderado (24\%) e artralgia/mialgia para grave (50\%). Desse modo, esperase que o estudo contribua para o despertar dos profissionais de saúde que atuam na unidade de saúde investigada, possibilitando um novo olhar para a saúde da mulher no climatério, numa perspectiva de estímulo ao autocuidado e a prevenção da saúde.

PALAVRAS-CHAVE: Sinais e Sintomas. Climatério. Enfermagem. Atenção Primária à Saúde.

Graduação em Enfermagem. Faculdade de Enfermagem Nova Esperança - FACENE. Departamento de Enfermagem.' CEP: 58067698, João Pessoa, Paraíba, Brasil.*Autor correspondente: ritacpeixoto@hotmail.com ORCID ID: 0000-0003-3011-5688; 0000-0003-4365-8358

Especialista em Urgência e Emergência e Unidade de Terapia Intensiva. Centro de Aperfeiçoamento Profissional -- II CEFAPP. CEP: 58030-021, João Pessoa, Paraíba, Brasil. ORCID ID: 0000-0002-0913-203X

Mestre em Saúde da Família. Faculdade de Enfermagem Nova Esperança - FACENE. Departamento de Enfermagem ${ }^{\text {III }}$ CEP: 58030-021, João Pessoa, Paraíba, Brasil. ORCID ID: 0000-0002-9336-7922

Mestre em Terapia Intensiva. Faculdade de Enfermagem Nova Esperança - FACENE. Departamento de Enfermagem. IV CEP: 58040-000, João Pessoa, Paraíba, Brasil. ORCID ID: 0000-0001-7642-2558

Doutora em Enfermagem. Faculdade de Enfermagem Nova Esperança - FACENE. Departamento de Enfermagem. IV CEP: 58067698, João Pessoa, Paraíba, Brasil. ORCID ID: 0000-0003-3008-2863

Submissão: 18/04/2019 Aceito: 05/02/20

DOI: 10.17695/revcsnevol18n1p18-25 


\section{INTRODUÇÃO}

O climatério é um fenômeno biológico da vida que se caracteriza pela transição entre a fase reprodutiva para não reprodutiva da mulher. Trata-se de um processo não patológico que ocorre em mulheres entre 40 e 65 anos de idade. ${ }^{1}$ É nesse período que acontece a menopausa, ou seja, a interrupção definitiva do fluxo menstrual nos últimos 12 meses. Este fenômeno consiste em uma consequência do declínio dos hormônios ovarianos (de forma natural), por intervenções cirúrgicas como a histerectomia ou ooforectomia (forma artificial). Quando associado às manifestações clínicas características da fase, é denominado como síndrome do climatério. ${ }^{2}$

Trata-se de uma fase natural da vida da mulher e, apesar de não ser considerado uma doença, traz um complexo de sinais e sintomas que podem comprometer o bem-estar geral e a qualidade de vida. ${ }^{3}$ Segundo o Manual de Atenção à Mulher no Climatério/Menopausa, as manifestações clínicas do climatério classificam-se em físicas e neuropsíquicas. Dentre as físicas temos os fogachos, com ou sem sudorese, alterações no ciclo menstrual, prolapsos genitais, incontinência urinária, dislipidemias, osteoporose e atrofias geniturinárias. Os sintomas neuropsíquicos ou neurovegetativos se caracterizam por insônia, irritabilidade, fadiga, ansiedade, melancolia e baixa autoestima. ${ }^{1}$

As modificações orgânicas vivenciadas pela mulher no climatério fazem surgir novos sentimentos, positivo e/ou negativos, e ambos envolvem perdas e ganhos que podem gerar crises e, sobretudo, possibilitar novas conquistas e mudanças na vida. ${ }^{4}$ Contudo, a assistência as mulheres no climatério pelo Sistema Único de Saúde (SUS), por meio das Unidades de Saúde da Família (USF's), pressupõe a existência de profissionais de saúde devidamente capacitados e sensibilizados para as particularidades inerentes a esse grupo específico, adotando uma abordagem clínica de caráter holístico por meio da escuta qualificada, acolhimento, incentivando o autocuidado e valorizando a participação ativa da clientela feminina na sua história de vida e de saúde. Sendo assim, as mulheres precisam ser empoderadas com informações para que tenham atitudes e sentimentos positivos em relação a esta etapa natural da vida..$^{1,5}$ Nesta direção, o papel dos membros da equipe das USF's é acolher, orientar, informar e educar adequadamente as mulheres, buscando prevenir e superar os desconfortos deste período como um todo, trazendo melhorias para a saúde da mulher. ${ }^{6}$

Neste contexto, com o intuito de trazer esclarecimentos à comunidade científica e aos profissionais de saúde, especialmente àqueles que atuam nas USF's, gerando subsídio para uma melhor compreensão a respeito do período do climatério, o presente estudo objetivou avaliar a sintomatologia vivenciada por mulheres no climatério atendidas na atenção primária, buscando compreender como estes sintomas podem interferir na qualidade de vida das mulheres.

\section{MATERIAL E MÉTODOS}

Pesquisa de caráter exploratório, descritiva, com abordagem quantitativa, realizada em uma Unidade da Estratégia Saúde da Família, situada no município de 
João Pessoa/PB. Participaram da pesquisa 50 mulheres no climatério, que atenderam ao único critério de inclusão que era ter idade entre 40 e 65 anos. Foram excluídas as mulheres que faziam reposição hormonal ou uso de fitoestrógenos, visto que essas substâncias interferem na sintomatologia, e as que estavam no climatério precoce (ou seja, antes dos 40 anos), histerectomizadas ou com a capacidade cognitiva prejudicada.

Os dados foram coletados nos meses de setembro e outubro de 2018 , seguindo as etapas: contato prévio com cada participante da pesquisa, quando foram explanados os objetivos e justificativa do estudo; explicação sobre a importância de participar da pesquisa e da assinatura do Termo de Consentimento Livre e Esclarecido, além de esclarecimentos quanto a garantia do anonimato. Por fim, aplicados os instrumentos para coleta de dados: I- Formulário de entrevista semiestruturado, com questões relacionadas ao perfil sociodemográfico das participantes do estudo e II - O Índice Menopausal de Blatt e Kuperman (IBMK), que é um instrumento já validado no Brasil, utilizado para especificar e classificar os sintomas do climatério. Os dados foram organizados em uma planilha do programa Excel 2010 e apresentados em tabelas, sendo posteriormente discutidos conforme a literatura pertinente.

O projeto foi aprovado pelo Comitê de Ética em Pesquisa-CEP da Faculdade de Enfermagem e de Medicina Nova Esperança FACENE/FAMENE, sob o Protocolo $n^{\circ}$ 143/2018 e CAAE: 94524318.3.0000.5179.

\section{RESULTADOS E DISCUSSÃO}

Foram entrevistadas mulheres entre 40 e 65 anos de idade, sendo $52 \%$ com idade inferior a 50 anos, $68 \%$ casadas, $44 \%$ frequentaram a escola durante 9 anos e 58\% com renda familiar de até dois salários mínimos (Tabela 1).

TABELA 1: Perfil sociodemográfico de mulheres atendidas em uma Unidade da Estratégia Saúde da Família, situada no município de João Pessoa/PB, Brasil, 2018

\begin{tabular}{ccc}
\hline \hline VARIÁVEL & N & Porcentagem (\%) \\
\hline Idade & & 52 \\
\hline 50 & 26 & 48 \\
\hline 50 & 24 & 68 \\
\hline \hline Estado Civil & & 32 \\
\hline Casada & 34 & \\
Solteira & 16 & 32 \\
Anos estudados & & 44 \\
\hline$<5$ & 16 & 24 \\
\hline 5-9 & 22 & \\
$\geq 10$ & 12 & 24 \\
Renda Familiar & & 58 \\
1 1 salário mínimo & 12 & 18 \\
1-2 salários mínimos & 29 & 100 \\
$\geq 3$ salários mínimos & 09 & Fonte: Pesquisa direta, nov./dez., 2018. \\
\hline TOTAL & 50 & \\
\hline
\end{tabular}

Rev. Ciênc. Saúde Nova Esperança. João Pessoa-PB. 2020; 18(1): 18-25 
A apresentação de sinais e sintomas no período do climatério é diferenciada para cada mulher, em geral essas manifestações surgem a partir dos 40 anos de idade, podendo influenciar no processo saúde doença dessas mulheres, acarretando problemas somáticos e emocionais no decorrer deste período.? Ainda de acordo com os autores supracitados, no período do climatérico, a sexualidade mostra-se como aspecto relevante na qualidade de vida.

As mulheres casadas no climatério referem maior grau de insatisfação sexual do que as solteiras e separadas com atividade sexual, devido às alterações hormonais que por sua vez influenciam na libido destas.

Observou-se que as mulheres entrevistadas estudaram em média 9 anos, o que reflete em um grau de instrução reduzido, provavelmente, pode constituir um fator que limite a adesão dos indivíduos aos cuidados de saúde, principalmente no que se refere a sintomatologia do climatério. ${ }^{8}$

Os dados, referentes a Figura 1, tratam da intensidade dos sintomas apresentados por mulheres no climatério a partir da classificação do Índice Menopausal de Blatt e Kuperman (IBMK).

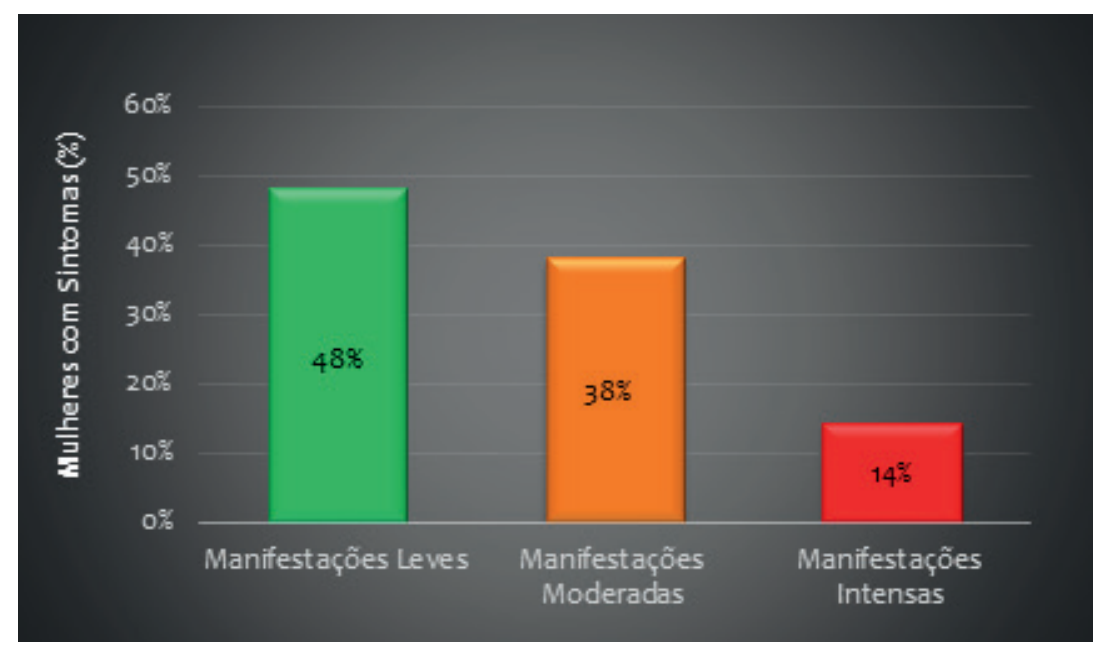

Figura 1: Distribuição das mulheres atendidas em uma Unidade da Estratégia Saúde da Família, segundo a intensidade dos sintomas a partir da classificação do Índice Menopausal de Blatt e Kuperman (IBMK). João Pessoa-PB, Brasil, 2018.

Em nosso estudo, observou-se que as mulheres entrevistadas $(n=50), 48 \%$ apresentaram manifestações leves, 38\% manifestações moderadas e apenas $14 \%$ apresentam manifestações intensas. Maior intensidade nos sintomas leves também foi observada em estudo realizado com 80 mulheres de 40 a 65 anos, pertencentes às Unidades de Saúde do Município de AlfenasMG. Além disso, considera-se que os sintomas leves estão associados a uma melhor qualidade de vida. ${ }^{9}$
Os sintomas que apresentaram graus de intensidade elevados foram: Artralgia/ Mialgia. Os graus moderados alcançaram percentuais em torno de $10 \%$ a $24 \%$, sendo mais elevado para cefaleia.

Dentre os sintomas vasomotores, o mais referido foram as palpitações, o que vai de acordo com os resultados encontrados em dois estudos realizados em cidades do Nordeste brasileiro, que encontraram $68,8 \%$ e $56,3 \%$ respectivamente. ${ }^{10}$ A terapia de reposição hormonal é capaz de reduzir em até 
$75 \%$ a intensidade deste sintoma. Neste estudo, as entrevistadas não fazem nenhum tipo de terapia de reposição hormonal, o que pode explicar a alta ocorrência encontrada para esta alteração vasomotora. ${ }^{11}$

A Tabela 2 expressa a frequência dos sintomas apresentados pelas mulheres, conforme a intensidade, segundo o IMBK.

TABELA 2: Distribuição da amostra, segundo a frequência e intensidade dos sintomas no período do climatério classificados pelo IMBK. João Pessoa-PB, Brasil, 2018

\begin{tabular}{|c|c|c|c|c|c|c|}
\hline \multirow{2}{*}{ VARIÁVEL } & \multicolumn{2}{|c|}{ LEVE } & \multicolumn{2}{|c|}{ MODERADO } & \multicolumn{2}{|c|}{ INTENSO } \\
\hline & $\mathbf{N}$ & $\%$ & $\mathbf{N}$ & $\%$ & $\mathbf{N}$ & $\%$ \\
\hline Calor ou fogachos & 9 & 18 & 5 & 10 & 12 & 24 \\
\hline Parestesia & 17 & 34 & 7 & 14 & 7 & 14 \\
\hline Insônia & 7 & 14 & 7 & 14 & 16 & 32 \\
\hline Nervosimo & 17 & 34 & 10 & 20 & 11 & 22 \\
\hline Melancolia & 12 & 24 & 9 & 18 & 13 & 26 \\
\hline Vertigem & 18 & 36 & 9 & 18 & 8 & 16 \\
\hline Fadiga & 16 & 32 & 6 & 12 & 6 & 12 \\
\hline Artralgia/mialgia & 7 & 14 & 11 & 22 & 25 & 50 \\
\hline Cefaleia & 11 & 22 & 12 & 24 & 10 & 20 \\
\hline Palpitações & 20 & 40 & 5 & 10 & 3 & 6 \\
\hline Formigamento & 16 & 32 & 8 & 16 & 5 & 10 \\
\hline
\end{tabular}

A cefaleia apresentou intensidade considerável, visto que, as manifestações típicas do climatério têm intensidades diversas variando de uma mulher para outra. Em um estudo transversal e quantitativo, realizado com 80 mulheres, no município de São Paulo, $37,5 \%$, apresentaram cefaleia. ${ }^{12} \mathrm{O}$ sintoma como a cefaleia pode estar associado a insônia visto que impossibilita um verdadeiro relaxamento do corpo, impactando negativamente na realização das atividades diárias da mulher. ${ }^{13}$

Já a artralgia/miagia é resultado da carência estrogênica que interfere na constituição dos tecidos e músculos, comprometendo a mobilidade física, agravando-se à medida que os anos se passam. ${ }^{1}$ Diante deste contexto, através de inquérito populacional envolvendo 303 mulheres na cidade de Maceió, onde foi avaliada a intensidade da sintomatologia do climatério, este mesmo sintoma (artralgia/mialgia) foi o segundo sintoma mais prevalente, observado em $81,85 \%$ das mulheres entrevistadas. ${ }^{14} \mathrm{Em}$ outro estudo transversal e descritivo realizado no município de Anápolis - GO, com 247 mulheres na pós-menopausa de 40 a 60 anos, constatou-se, também, a presença da artralgia/ mialgia em torno de $77,7 \%$, de forma severa. ${ }^{15}$

Diante da complexidade da sintomatologia do climatério, no sentido de trazer benefícios para a saúde das mulheres nesta fase, estudos comprovam a eficácia da atividade física na melhora destes sintomas e na qualidade de vida. Em revisão sistemática sobre a qualidade de vida e a importância da atividade física na melhora dos sintomas do climatério, constataram através de análise de 11 artigos científicos, a relevância dos exercícios físicos neste momento de transição feminina. ${ }^{16}$ 
Diante do exposto, as manifestações apresentadas pelo grupo pesquisado têm como principal etiologia o hipoestrogenismo que ocorre durante o climatério, interferindo significativamente em diversos sistemas do organismo da mulher, gerando alterações no funcionamento e qualidade de vida. Ressaltase ainda que os sintomas evidenciados pelas participantes deste estudo carregam

\section{CONCLUSÕES}

A partir desse estudo, conclui-se que em relação a sintomatologia, um pouco menos da metade das mulheres apresentou sintomas de intensidade leve.

Os resultados aqui demonstrados denunciam a necessidade de um programa de educação continuada com os profissionais de saúde, com vistas a melhorar e aperfeiçoar a assistência à saúde da mulher no climatério. significados psicossociais e culturais, inerentes a esse período, os quais devem ser considerados. ${ }^{17}$ Por esta razão, torna-se imperativo a prestação de assistência integral, holística e multidimensional no sentido de promover às mulheres condições para perpassarem o climatério da forma menos traumática possível. ${ }^{1,9}$
Sugere-se, também, a criação de grupos educativos - terapêuticos voltados às mulheres no climatério, para que estas sejam conscientizadas sobre a possibilidade de surgimento da síndrome, de modo que a mulher entenda que, apesar disso, trata-se de uma fase natural do ciclo de vida feminino e não um processo patológico.

\title{
CLIMATTERY: SYMPTOMS IN WOMEN ATTENDED IN PRIMARY CARE
}

\begin{abstract}
The climacteric is a period experienced during the woman's lifecycle, accompanied by a set of physiological, social, and psychological changes. It represents the milestone of female aging, significantly influencing quality of life as it has a complex of specific signs and symptoms. The research aimed to identify the symptoms experienced by women during the climacteric period, attended in primary care. For this, an exploratory and descriptive study was carried out, with a quantitative approach, performed in a Family Health Strategy Unit, in the city of João Pessoa / PB, taking as sample 50 women aged between 40 and 65 years. The sociodemographic characteristics were evaluated and the Blatt \& Kuperman Menopausal Index (IBMK) was used, which is able to identify the signs and symptoms of the climacteric and classify them according to the intensity: light, moderate and intense. It was concluded that, of the women interviewed, $52 \%$ were under 50 years old and $44 \%$ attended school for 9 years. Regarding the intensity of climacteric symptoms, it is clear that $48 \%$ had mild manifestations. There was a predominance of palpitations for mild (40\%), headache for moderate (24\%) and arthralgia / myalgia for severe (50\%). Thus, it is expected that the study will contribute to the awakening of health professionals who work in the health unit investigated, enabling a new look at women's health in the climacteric, in a perspective of encouraging self-care and health prevention.
\end{abstract}

KEYWORDS: Signals and Symptoms. Climacteric. Nursing. Primary Health Care. 


\section{REFERÊNCIAS}

1. Ministério da Saúde (BR). Manual de Atenção à Mulher no Climatério/Menopausa. 2008. Brasília: Ministério da Saúde; 2015.

2. Alves ERP, Costa AM, Bezerra SMMS, Nakano MAS, Cavalcanti AMTS, Dias MD. Climatério: a intensidade dos sintomas e o desempenho sexual. Texto Contexto Enferm. 2015; 24(1): 64-7.

3. Souza NLSA, Araújo CLO. Marco do envelhecimento feminino, a menopausa: sua vivência, em uma revisão de literatura. Revista Kairós Gerontologia. 2015; 18(2): 149-65.

4. Silva JPL, Marcolino EC, Fernandes NMS, Moura IM, Araújo MZ. Representações do climatério e suas repercussões na vida da mulher: uma revisão sistemática. In: Anais $4^{\circ}$ Congresso de EnveIhecimento Humano. 2015; 2(1).

5. Oliveira SRG, Wendhausen ALP. (Re) significando a educação em saúde: dificuldades e possibilidades da Estratégia Saúde da Família. Trab. Educ. Saúde. 2014; 12(1): 129-47.

6. Candeia SMAS, et al. Conhecimento de Um Grupo de Mulheres Sobre o Climatério.

Revista Temas em Saúde. 2013; 13(1): 42-46.

7. Santos AAS, Silva FV, Martins FL. Percepção das mulheres no município de Paulo Afonso, na Bahia, sobre as mudanças corporais e emocionais no período do climatério. Estação Científica. 2016; 6(1): 91-103.

8. Oliveira TM, Valdez FML, Lima KES, MagaIhães MS, Abdon APV, Bezerra IN. Prevalência de Incontinência Urinária e Fatores Associados em Mulheres no Climatério em uma Unidade de Atenção Primária à Saúde. Rev. Bras. Promoç.
Saúde. 2015; 28(4): 606-12.

9. Ribeiro AS, Soares AKA, Siqueira VMS, Souza WA, Podestá MHMC, Ferreira EB. Avaliação dos sintomas e da qualidade de vida das mulheres no climatério. Revista da Universidade Vale do Rio Verde. 2015; 13(1): 46-65.

10. Brito LMO, Chein MBC, Correa RGCF, Castro MS, Cutrim PT, Machado GM. Ocorrência de Sintomas Clínicos em Mulheres Climatéricas Assistidas em um Serviço de Referência em São Luís/ Maranhão - Symptoms of Occurrence in Clinical Weather Women in São Luís/Maranhão-Brazil. Revista de Pesquisa em Saúde. 2016; 17(2).

11. Pardini D. Terapia de reposição hormonal na menopausa. Arq. Brasileiros de Endocrinologia \& Metabologia. 2014; 58: 172-81.

12. Melo CRM, Reis ES, Silva LCFP, Sola EPS, Chofakian CBN. Aplicação do Índice Menopausal de Kupperman: um estudo transversal com mulheres climatéricas. Espaço para a saúde - Revista de Saúde Pública do Paraná. 2016; 17(2): 41-50.

13. Correa KM, Bittencourt LRA, Tufik S, Hachul $H$. Frequência dos transtornos de sono em muIheres na pós-menopausa com sobrepeso / obesidade. Rev. Bras. Ginecol. Obstet. 2014; 36(2): 90-96.

14. Araújo JBS, Santos GC, Nascimento MA, Dantas JSG, Ribeiro ASC. Avaliação da intensidade da sintomatologia do climatério em mulheres: inquérito populacional na cidade de Maceió, Alagoas. Ciências Biológicas e da Saúde. 2015; 2(3): 101-11.

15. Santos TR, Pereira SVM, Santos RL. Intensida 
revista de NOVA
ciências

daênciúd ESPERANÇA

de da sintomatologia climatérica em mulheres pós - menopausa. Ver. Rene. 2016; 17(2): 225-32.

16. Fretta TB, Reis NM, Machado Z, Guimarães ACA. Melhora dos sintomas do climatério por meio da atividade física: uma revisão sistemáti- ca. Saúde Rev. 2017; 17(46): 67-78.

17. Veloso LC, Nery IS, Celestino DSS. Mudanças biopsicossociais vivenciadas por mulheres no climatério: um reflexo na influência de gênero. Rev. Saúde em Foco. 2014; 1(1): 46-71. 